\title{
An inquiry into the certification potential of built environments' affordance
}

\author{
Ardeshir Mahdavi* and Christiane Berger \\ Department of Building Physics and Building Ecology, TU Wien, Vienna, Austria
}

\begin{abstract}
Buildings are typically equipped with a number of elements and devices (e.g., windows, blinds, luminaires, radiators) to control indoor environmental conditions. The availability, effectiveness, and usability of control devices and their interfaces constitute an essential aspect of built environments' quality. This aspect has been sometimes referred to as indoor-environmental "affordance" (or "ecological valency") and can be interpreted as a descriptor of buildings' responsiveness toward inhabitants' needs and requirements. However, in contrast to some other building quality aspects such as energy efficiency, there is a lack of systematic evaluation or certifications procedures for objective characterization of indoor environments' affordance as related to the availability and effectiveness of control devices and their human interfaces. This paper entails an exploration of the potential for and associated challenges of designing and implementing affordance measurement and certification procedures. Challenges include, above all, assigning relative weights and coefficient values to different domains (e.g., thermal, visual, acoustical), devices, and levels of their effectiveness. Irrespective of their success to date, attempts toward operationalization of indoor-environmental affordance can be quite beneficial: The affordance framework can offer a systematic, intuitive, and compact framework to guide the workflow toward the provision and maintenance of adequate indoor environments for human occupancy.
\end{abstract}

\section{Introduction}

Buildings are typically equipped with a number of elements and devices to control indoor environmental conditions. Examples are windows, blinds, luminaires, radiators, and fans. Inhabitants of so-called free-running buildings or those with hybrid environmental control systems are commonly provided with various types of interfaces to operate such devices. The availability, effectiveness, and usability of control devices and their human interfaces constitute arguably an essential aspect of built environments quality and performance. This aspect has been sometimes referred to as (indoorenvironmentally relevant) "affordance" $[1,2]$, or - in human-ecological terminology - as "ecological valency" $[3,4,5]$. In this context, affordance can be thus interpreted as a descriptor of buildings' responsiveness toward inhabitants' needs and requirements. However, in contrast to some other building performance attributes such as energy efficiency, there is a lack of systematic evaluation or certifications procedures for objective characterization of indoor environments' affordance as related to the availability and effectiveness of control devices and their human interfaces.

In this context, it seems reasonable to ask if other essential quality aspects of buildings could be subjected to a kind of objective assessment resulting in respective certificates.
The present contribution expands on the work described in a previous paper [6] towards the exploration of the potential for designing and implementing affordance measurement and certification procedures, possibly involving detailed mathematical formulations or simplified rating systems.

\section{Theoretical foundations}

\subsection{Introductory remark}

The aforementioned outlined inquiry can be conceptually supported by prior work in multiple traditions, including the Vienna School of Human Ecology and the Ecological Psychology. Whereas the former tradition may provide a more consistent set of concepts, the latter is more widely known. The following sections (2.2 and 2.3) provide thus a brief reference to both of these traditions.

\subsection{Ecological psychology and the concept of affordance}

The interactions between people and their surrounding environment has been a central theme in cognitive and environmental psychology. An influential line of inquiry in this area goes back to the psychologist Gibson in general and his concept of "affordance" in particular [2]. In Gibson's diction, "affordances of the environment are what it offers the animal, what it provides or furnishes"

* Corresponding author: amahdavi@tuwien.ac.at 
[1]. Affordance, as Gibson seems to define it, is not dependent of a specific individual's recognition of it. But it can be recognized by individuals according to their needs. Moreover, perceiving affordances is connected with initiating actions. As such, people tend to intervene in their surroundings and modify affordances, such that they better match their needs. The conceptual background of Gibson's work display similarities to views formulated earlier by Uexküll [7]. However, the latter does not postulate the existence of affordances independent of their representations ("Umwelt").

The concept of affordance has been used in other fields such as human-computer-interaction and industrial design [8]. Hence, it can be also applied to environmental and architectural domains. Thereby, building design decisions and actual interventions (including control actions) in indoor environments could be suggested to enhance the respective repertoire of affordances.

\subsection{Human ecology and the concept of ecological valency}

Human ecology is a promising instance of a theory, that can guide the efforts toward indoor environmental quality assessment. As a scientific discipline, ecology deals with the relationships between living organisms and their surrounding world. Accordingly, human ecology may be simply defined as the ecology of the Homo sapiens. There are many traditions and associated approaches to human ecology. From the standpoint of the "Vienna School of Human Ecology" [3, 4, 5, 9], the construction and operation of buildings can be viewed as an integral part of the totality of largely regulatory operations initiated by human beings as they interact with their surrounding world. Human ecology offers a useful way of thinking about these interactions via a number of concepts, including the following pair:

i. the human beings' ecological potency;

ii. the surrounding world's ecological valency $[3,10]$.

Thereby, ecology potency refers to people's capability to cope and interact with the surrounding world. Ecological valency, on the other hand, denotes the totality of that surrounding world's characteristics (resources, opportunities, challenges, risks, hazards) as it relates to people's ecological potency. The concept of ecological valency was essentially dealt with in [7] and is also akin to Gibson's affordance $[1,2]$.

Given this conceptual framework, we can describe the main consideration in human ecology as the complex and dynamic relationships between the ecological potency of human beings and the ecological valency of their surrounding world. Human ecologically speaking, buildings are constructed and maintained with the intention to favourably influence the relationship between people's ecological potency and the ecological valency of their surrounding world. An instance of such intention expresses itself, for example, in the "shelter function" of the vernacular architecture [11]. Today, this intention is often formally expressed in terms of specifications of desirable indoor environmental conditions.

Note that assessing the ecological valency of the built environment cannot be fully achieved without taking inhabitants' ecological potency and its relationship to a given environment's ecological valency into account. Toward this end, a second pair of human ecological concepts can be useful, which concerns distinct aspects that can be attributed to the relationships between people and their surroundings, namely the material-energetic aspect and the information-related aspect of the potencyvalency relationships $[3,5,9]$. The relevance of this pair of concepts to the ecological valency assessment can be illustrated as follows. Such an assessment must not only consider objective indoor environmental states variables, but also people's subjective experiences and opinions. Thus, both material-energetic and information-related aspects of the relationships between inhabitants and the built environment must be taken into consideration.

\section{Limits of the operationalization potential of the built environments' affordance}

Given the preceding discourse of theoretical foundations in human ecology and ecological psychology, the problem of building quality assessment regarding inhabitants' control opportunities in indoor environments can be suggested to involve multiple challenges. First, how are we to simultaneously address the variance of inhabitants' ecological potency while we attempt to assess indoor environments' affordance? The former is arguably a population characteristic. A common approach in comparable circumstances (e.g., definition of thermal comfort requirements in buildings) is to assume a kind of "average" building user (typically, healthy adults) as the reference. This approach is obviously less than satisfactory, given the increased level of awareness concerning the importance and necessity of Universal Design principles. Nonetheless, even conventional comfort standards consider population variance in comfort requirements to a certain - albeit basic - degree, for instance via consideration of different building typologies (e.g., hospitals, schools, offices) and space use categories (e.g., operations room, corridor, lobby).

However, a more consequential coverage of the diversity of inhabitants' ecological potency would be desirable, as indoor environment's affordance should ideally accommodate people's diverse spectrum of ecological potencies. Nevertheless, we can at least partially justify the present contribution's concentration on affordance and its operationalization with the following argument: Improving an environment's affordance is associated with its capacity to offer a wide and flexible range of conditions. Enrichment of affordance is thus likely to benefit all occupants, irrespective of their diversity of their needs and capabilities. 
To further pursue the operationalization potential of the affordance concept, it may be useful to agree upon an adequate unit of observation. Assuming a number of background factors such as the climatic context and the building type, individual spaces (rooms) in a target building may be considered to be proper candidates for determination of affordance levels (see Figure 1). Even though the definition of discrete rooms and their function is not straightforward in all cases, most professionals and occupants have a fairly clear idea of the meaning of the concept. Specifically, maintaining desirable indoor environmental conditions is frequently practiced at the room level.

Given rooms as units of observation, we can further reflect on various aspects (or dimensions) of the affordance. In other words, we can discuss the conceptual space of affordance (see Figure 1).

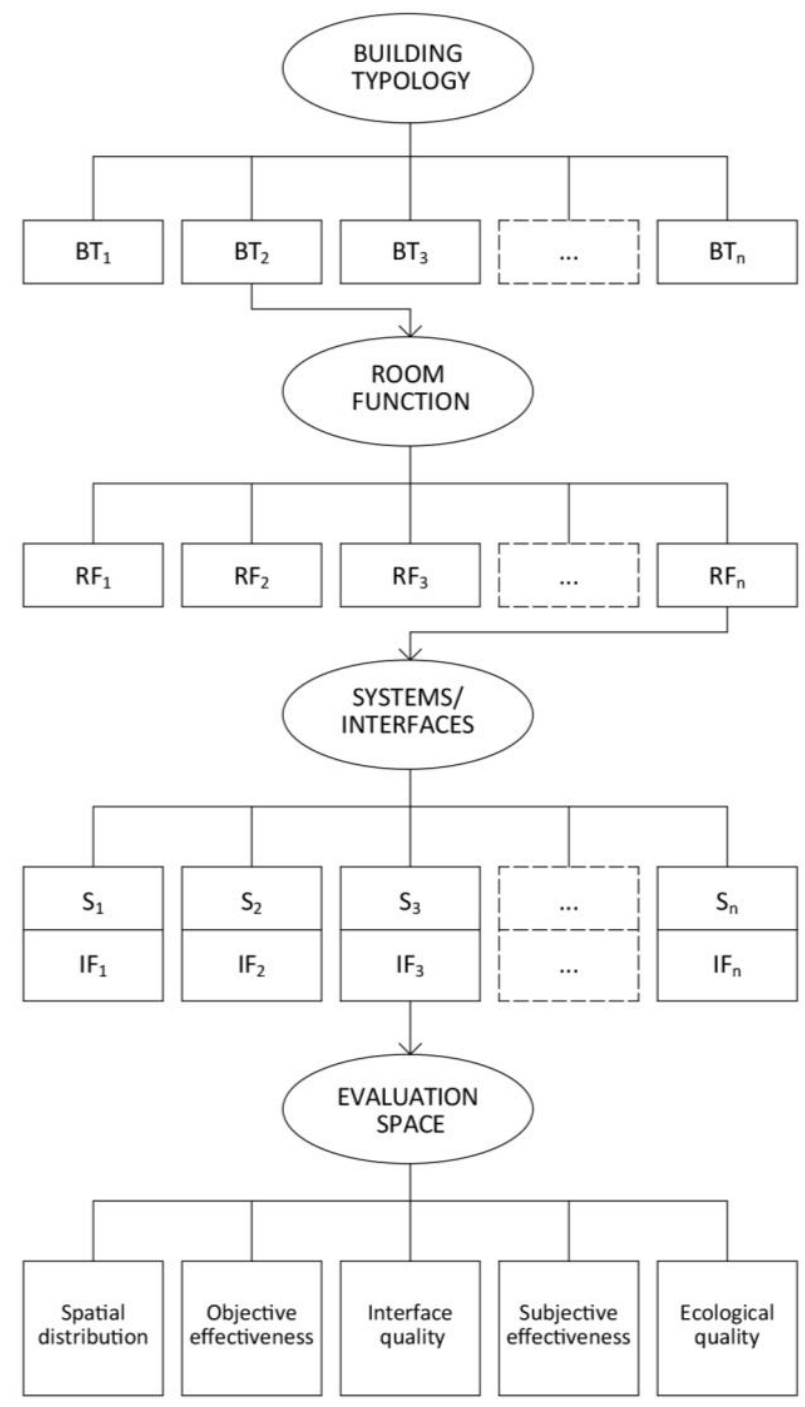

Fig. 1. Illustration of the underlying conceptual structure of a potential building affordance certification scheme. Starting from typological classification of buildings and functional classification of spaces, the latter are identified as the appropriate units of observation for control device benchmarking in multiple domains. Devices are suggested to be assessed according to five evaluation criteria.
Hereby, a natural starting point would be those properties of the indoor environment whose dynamic adjustability by inhabitants is desirable/necessary. For the purpose of the present discussion, consider variables pertaining to:

- hygro-thermal environment (temperature humidity),

- air quality (fresh air volume flow),

- visual environment (daylight, electrical lighting).

In a first approximation, the problem of affordance characterization (for instance, in terms of an "Affordance-Index") may be reduced to the availability and attributes of devices that enable inhabitants to control the relevant environmental variables. The said devices typically facilitate the modulation of mass and energy supplied to (or extracted from) a space (see Table 1). They may also change the distribution and composition of mass and energy distribution in the indoor environment. For instance, as a control device, a window can modulate the magnitude of fresh air volume flow into a room and influence indoor environmental variables such as air temperature and humidity. It can also influence the concentration of pollutants and thus the air quality. A shading device such as an external blind can modulate the magnitude of transmitted solar radiation and daylight, thus influencing indoor environmental conditions in view of the temporal and spatial distribution of illuminance and luminance levels.

Control devices in a space can be thought of the constituents of its affordance. Operationalization of affordance must thus involve the appraisal of the availability and quality of these devices. Different criteria may be take into consideration toward quality evaluation of control devices and their interfaces. A number of such criteria are suggest below, formulated in terms of five sets of questions (see Figure 2). Note that they are not claimed to be either the only or the most conclusive criteria:

- What is the spatial resolution level of the target zones of the control device? Can users control the state of their immediate surroundings?

- What is the degree of the objective effectiveness of the control device, i.e., can it fulfil, in a timely and sufficient manner, the intended task?

- Can the operation of the device be considered efficient in the sense of energy use and environmental impact?

- Can the device be deployed in a convenient and intuitive manner, or, in other words, does it come with an adequate user interface?

- What is the degree of the subjective effectiveness of the control device, i.e., do the users have the impression that it satisfactorily performs the intended functionality? 
Table 1. An illustrative taxonomy of buildings' assorted control devices together with associated mechanisms (processes) they employ to influence indoor environmental conditions. " $\checkmark "$ stands for the main process mode, "O" stands for the secondary process mode (or side effect), and "-" for no impact.

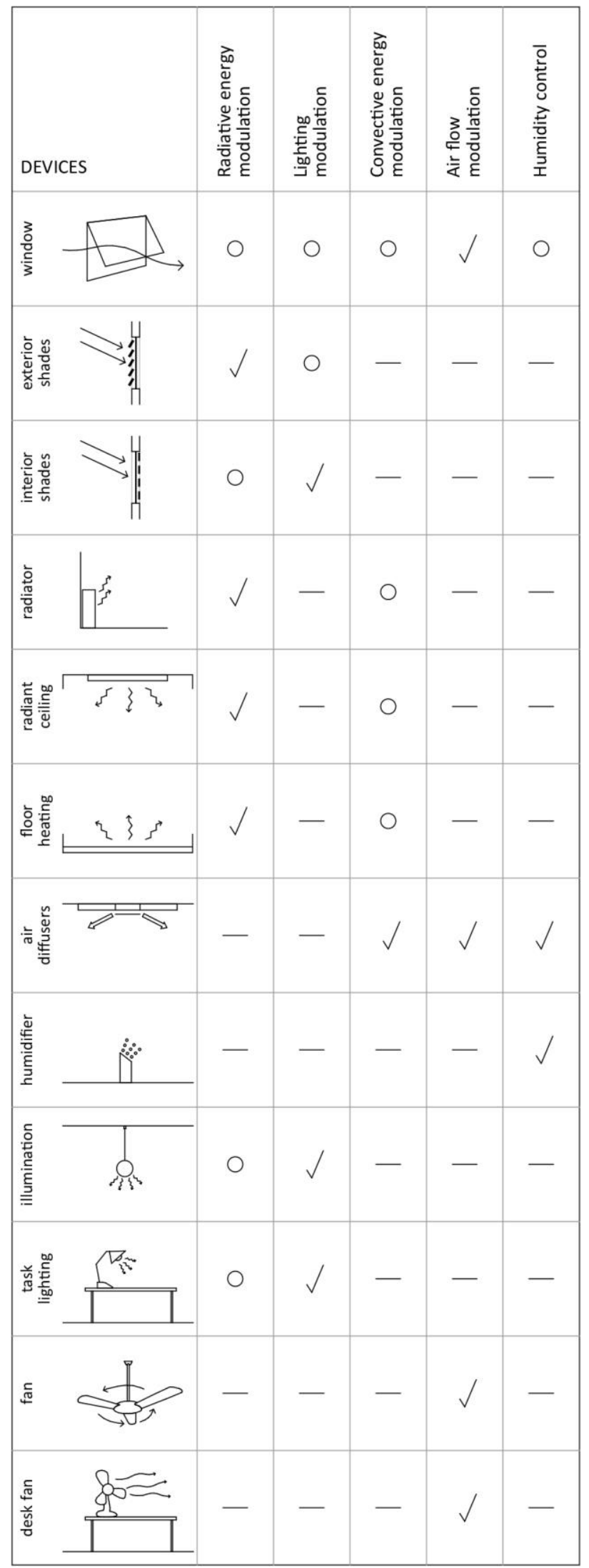

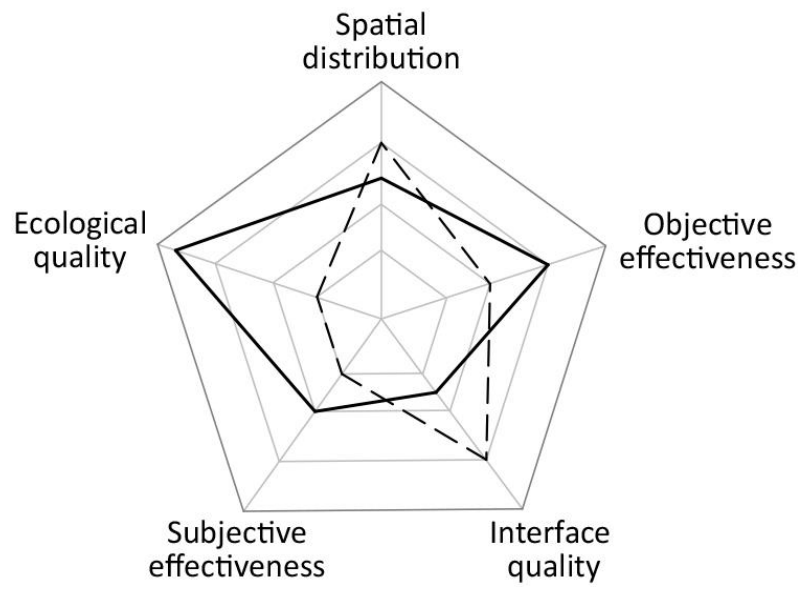

Fig. 2. An illustrative polar diagram with evaluation results of environmental control devices (two domains) in a space in terms of five evaluation criteria (see text)

Following this line of inquiry, the following path to the operationalization of the indoor-environmental affordance of a specific built space emerges. For each constituent domain of indoor climate (i.e., thermal, visual, air quality), an integral function over all available devices in that domain would have be derived, whereby weights for the aforementioned quality criteria (spatial resolution, objective effectiveness, environmental impact, user interface, subjective effectiveness) would have to be assigned. Subsequently, another weighting function would be needed to integrate affordance indices of the different domains.

Following the above sketched path involves a number of challenges. However, the biggest challenge might not be so much the formulation of a general mathematical formalism, e.g., a set of equations to calculate numeric values of the affordance index (AI). Rather, the main problem lies in the attribution of numeric values (or points) not only to the device criteria variables, but also to the weighting factors needed to arrive at integrated numeric values for a practically applicable general AI. In fact, weights are not only needed to integrate over AI values of different devices in a specific space, but also to integrate AI values of different spaces in a building. This means that weighting factors would be required for integration of device level $\mathrm{AI}$ values $\left(\mathrm{AI}_{\mathrm{D}}\right)$ into space level values $\left(\mathrm{AI}_{\mathrm{S}}\right)$, and space level values into building level values $\left(\mathrm{AI}_{\mathrm{B}}\right)$.

To illustrate this challenge, consider the derivation of $\mathrm{AI}$ for one device $\left(A I_{D i}\right)$ out of $n$ devices in one space $\left(\mathrm{S}_{\mathrm{j}}\right)$ out of $k$ spaces in a building (B). Assuming this device can obtain, for each quality criterion (e.g., C1 to C5), a certain number of points (e.g., 0 to 10) and treating these points with respective weights $\left(\mathrm{W}_{\mathrm{C} 1}\right.$ to $\mathrm{W}_{\mathrm{C} 5}$ totaling to 1), we obtain:

$\mathrm{AI}_{\mathrm{Di}}=\mathrm{AI}_{\mathrm{C} 1} \cdot \mathrm{W}_{\mathrm{C} 1}+\mathrm{AI}_{\mathrm{C} 2} \cdot \mathrm{W}_{\mathrm{C} 2}+\ldots+\mathrm{AI}_{\mathrm{C} 5} \cdot \mathrm{W}_{\mathrm{C} 5}$ 
To further derive the affordance of the space $\mathrm{Sj}$, a weighted sum of individual devices in this space must be calculated:

$$
\mathrm{AI}_{\mathrm{Sj}}=\mathrm{AI}_{\mathrm{D} 1} \cdot \mathrm{W}_{\mathrm{D} 1}+\mathrm{AI}_{\mathrm{D} 2} \cdot \mathrm{W}_{\mathrm{D} 2}+\ldots+\mathrm{AI}_{\mathrm{Dn}} \cdot \mathrm{W}_{\mathrm{Dn}}
$$

Finally, the building's affordance index $\left(\mathrm{AI}_{\mathrm{B}}\right)$ would have to be derived from the weighted sum of the affordance indices of all building's spaces:

$\mathrm{AI}_{\mathrm{B}}=\mathrm{AI}_{\mathrm{S} 1} \cdot \mathrm{W}_{\mathrm{S} 1}+\mathrm{AI}_{\mathrm{S} 2} \cdot \mathrm{W}_{\mathrm{S} 2}+\ldots+\mathrm{AI}_{\mathrm{Sk}} \cdot \mathrm{W}_{\mathrm{Sk}}$

It goes without saying that there are considerable inherent uncertainties and complexities involved in the process of arriving at such a large number of weighting factors pertaining to evaluation criteria of individual devices (i.e., $\mathrm{W}_{\mathrm{C} 1}$ to $\mathrm{W}_{\mathrm{C} 5}$ ), consideration of multiple devices in a space (i.e., $\mathrm{W}_{\mathrm{D} 1}$ to $\mathrm{W}_{\mathrm{Dn}}$ ), and aggregation over multiple spaces (i.e., $\mathrm{W}_{\mathrm{S} 1}$ to $\mathrm{W}_{\mathrm{Sk}}$ ). Consequently, defining and implementing practical procedures for the respective computation of AI values would be in most cases difficult, if not untenable. Practitioners face many challenges, even in the considerably simpler case of energy-specific certification documents. A circumstance that explains the frequently insufficient degree of such certifications' reproducibility. Consequently, it is highly doubtful that independent instances computing AI values for a given building (or even a single space in a building) would arrive at similar numeric values.

As an alternative to a detailed and conceptually rigorous approach to the computation of numeric AI values, one might consider the utilization of a kind of point-based rating system, similar to those deployed in common building quality rating and certification systems. Presumably, the availability, number, zonal distribution, objective and subjective effectiveness, user interface quality, and environmental efficiency could be captured via a simplified procedure, whereby (weighted) points would be assigned to each item and accumulated to arrive at overall scores or rankings. Such an approach may involve some benefits in terms of practical applicability. However, aside from being unsatisfactory from the intellectual point of view, it would not necessarily lead to a robust method resulting in reproducible and meaningful quality assessments.

It has been argued in the past, specifically in the context of common environmental quality rating systems for buildings, that there is more to the utility of rating systems than their computational precision and numeric reproducibility. Rather, so the argument goes, the primary value of these rating procedures is in their role in raising the general awareness of professionals and public concerning buildings' quality and environmental impact. Even the takeover of environmental rating systems for marketing purposes has been suggested to involve, on balance, more positive ramifications than negative ones. This argument could be presumably applied also in case of affordance certification potential explored in the present contribution. Such an argument cannot be entirely refuted here, given the absence of sufficiently conclusive evidence. But even if true, it would not make, from a consistent scientific point of view, a convincing case in favour of an unsubstantiated building affordance certification procedure.

\section{Conclusion}

This contribution explored the possibility of systematically defining indoor environments' affordance. If feasible, an operationalized affordance index would provide a useful indicator of the quality of a building in view of the degree to which it would accommodate inhabitants' needs and requirements. The brief investigation offered in the present contribution suggests that, from a theoretical standpoint, it is conceivable to realize such a possibility and devise associated procedures. However, this would not be a trivial undertaking, as it involves multiple uncertainties. For one thing, it would be necessary to consider the considerable diversity of buildings' occupants and the range and complexity of their needs, expectations, and capabilities. Furthermore, multiple domains of indoor environmental services (e.g., thermal, visual, acoustical, air quality) and the associated physical components (control devices) would have to be considered, together with their numbers, their spatial distribution, their subjective and objective effectiveness, and their performance in terms of energy use and environmental impact.

To formalize a numerical affordance index, both mathematical formulas or simpler rating systems (e.g., point-based evaluation methods) could be conceived. However, efforts in this direction would have to address a number of challenges, including the difficult and arguably subjective - if not arbitrary - process of assigning relative weights and coefficient values to different building types, different room functions, different environmental control domains, different devices, and different levels of effectiveness and usability. Such difficulties could render the outcome of the above mentioned affordance determination procedures non-reproducible and thus limited in expressiveness and usefulness. This, however, does not imply that that attempts toward the definition of affordance indicators and derivation of their values are futile. Concepts in human ecology (such as ecological potency and ecological valency) and ecological psychology (i.e., affordance) have the potential to guide the approaches and sharpen the attitudes of the relevant professionals in building design and operation phases. Accordingly, ongoing efforts are attempting to test the usability of a preliminary affordance assessment protocol and explore the reproducibility of its deployment results [12]. Even if this effort or similar undertakings shall not yield reproducible and rigorous numeric benchmarks, the related concepts and their underlying theories could still provide a systematic and compact conceptual map to situate and organize planning and operational objectives as relevant to provision and maintenance of adequate indoor environments for human occupancy. 
Acknowledgement: The treatment presented in this paper benefited from authors' participation in the IEA EBC Annex 79 and the related discussions.

\section{References}

1. J. Gibson. The Ecological Approach to Visual Perception. ISBN 0-89859-959-8 (1979)

2. J. Gibson. The Theory of Affordances. Perceiving, Acting, and Knowing, Edited by Robert Shaw and John Bransford. ISBN 0-470-99014-7 (1977)

3. H. Knötig. Human Ecology - The exact science of the interrelationships between Homo sapiens and the outside world surrounding this living and thinking being. The sixth meeting of the Society for Human Ecology "Human Ecology: Crossing Boundaries". Snowbird, Utah, USA (1992)

4. H. Knötig. Some essentials of the Vienna School of Human Ecology. Proceedings of the 1992 Birmingham Symposium; Austrian and British Efforts in Human Ecology. Archivum Oecologiae Hominis. Vienna, Austria (1992)

5. A. Mahdavi. The human factor in sustainable architecture. Low Energy Low Carbon Architecture: Recent Advances \& Future Directions (Sustainable Energy Developments). Edited by Khaled A. AlSallal; Taylor \& Francis, London, UK. pp. 137 158 (2016)

6. A. Mahdavi. Can we quantify the ecological valency of built environments? 12th Envibuild conference (2017) - Buildings and Environment - From Research to Application "enviBUILD - Proceedings: Building and Environment", Trans Tech Publications. ISBN: 978-3-0357-1202-5 (2019)

7. J. Uexküll. Kompositionslehre der Natur. Edited by Thure von Uexküll. Frankfurt am Main, Germany (1920)

8. D. Norman. The design of everyday things. ISBN 9780465050659 (2013)

9. A. Mahdavi. Approaches to Noise Control: A Human Ecological Perspective. Proceedings of the NOISE-CON 96 (The 1996 National Conference on Noise Control Engineering). Bellevue, WA, USA. pp. $649-654$ (1996)

10. A. Mahdavi. Human Ecological Reflections on the Architecture of the "Well-tempered Environment". Proceedings of the 1996 International Symposium of CIB W67 (Energy and Mass Flows in the Life Cycle of Buildings). Vienna, Austria. pp. 11 - 22 (1996)

11. A. Mahdavi. A Human Ecological View of "Traditional" Architecture. Human Ecology Review (HER). Volume 3, Number 1. pp. 108 - 114 (1996)

12. A. Mahdavi, H. Teufl, and C. Berger. Application of the Ecological Valency concept to buildings' environmental control systems. Proceedings of IAQVEC 2019 (to be published) 